\title{
Construction of a Synthetically Engineered nirB Promoter for Expression of Recombinant Protein in Escherichia coli
}

\author{
Reza Nasr ${ }^{1}$; Mohammad Reza Akbari Eidgahi ${ }^{1, *}$ \\ ${ }^{1}$ Semnan Biotechnology Research Center, Semnan University of Medical Sciences, Semnan, IR Iran \\ ${ }^{*}$ Corresponding author: Mohammad Reza Akbari Eidgahi, Semnan Biotechnology Research Center, Semnan University of Medical Sciences, Semnan, IR Iran. Tel/Fax: +98-2313354187, \\ E-mail:mrakbari201177@yahoo.com
}

Received: December 18, 2013; Revised: May 5, 2014; Accepted: May 7, 2014

\begin{abstract}
Background: Anaerobic-inducible promoters are alternatives of chemical-inducible promoters for expression of recombinant proteins especially in conditions where chemical induction is not possible or anaerobic conditions are preferable. The nirB promoter is the promoter of the first gene of nir operon in Escherichia coli, which encodes NADH-dependent nitrite reductase. This promoter is naturally induced under anaerobic conditions and upregulated by nitrite and nitrate.

Objectives: The current study was carried out to construct a synthetic nirB promoter that does not respond to chemical inducers (nitrite or nitrate), but instead responds to anaerobic induction. For this purpose, a new plasmid was constructed (pFSnirB78-23LTB), which contains a synthetic nirB promoter. The activity of this plasmid was evaluated in E. coli under both aerobic and anaerobic conditions and in response to chemical inducers, nitrite and nitrate.

Materials and Methods: A synthetic nirB promoter was firstly cloned into a pKK223 derivative plasmid and then the heat labile toxin B subunit gene (LTB) of entrotoxigenic E. coli was cloned under the control of this promoter. The inducibility of this plasmid in E. coli was measured under anaerobic conditions in the presence or absence of nitrite or nitrate by ganglioside GM1 ELISA.

Results: Our data showed that this promoter is strongly induced under anaerobic conditions while it showed much lower activity (11\%) under aerobic conditions. In contrast to the native promoter, this promoter was not induced by chemical inducers, nitrite or nitrate.

Conclusions: This study showed that the recombinant protein produced under the control of synthetic nirB promoter has critical characteristics such as pentamer formation, receptor recognition ability and conservation of antigenic epitopes. In addition, the data showed anaerobiosis and chemical inducers had no adverse effects on recombinant proteins. Based on the results, this synthetic promoter is suitable for use in live delivery vaccines or drug systems and for production of recombinant proteins especially oxygen sensitive proteins.
\end{abstract}

Keywords:Recombinant Proteins; Enzyme-linked Immunosorbent Assay; Escherichia coli; Nitrate reductase (NADH); Nitrite Reductase $(\mathrm{NAD}(\mathrm{P}) \mathrm{H})$; Gene Expression

\section{Background}

Expression of recombinant proteins in Escherichia coli are usually performed in high oxygen tension and controlled by various chemically or physiologically inducible promoters $(1,2)$. Chemically inducible promoters such as lac, tac, trc, trp and $T 7$, used routinely for production of recombinant proteins but they have economic and technical restrictions particularly when used for production on an industrial scale (3). They may require additional steps in the production process and the isolation of the inducer from the final product is costly. On the other hand, in case of oxygen-sensitive proteins, high $\mathrm{O}_{2}$ tension may have adverse effects on protein structure and function. Hence, many alternative inducible and controllable promoter systems have been investigated for recombinant protein production $(3,4)$.

As transcription is basically controlled via promoter elements, promoter engineering is a fundamental factor for control of transcription and thereupon protein produc- tion (5). Anaerobically inducible promoters are an alternative of chemically inducible promoters for expression of recombinant proteins especially in conditions where chemical induction is impossible or anaerobic conditions are preferable (6). The nirB promoter is the promoter of the first gene of E. coli NADH-dependent nitrite reductase operon. This promoter is induced in anaerobic conditions and up regulated in response to nitrite and nitrate.

There are two critical regions on this promoter for induction by anaerobic conditions. The first being a hexamer region, TAAGGT, at -10 positions that it is necessary for anaerobic activation and mutation in this region may result in loss of promoter activity. The second region is the fumarate and nitrate reductase regulatory (FNR) protein-binding site and mutation in this region results in decreasing promoter induction. FNR protein encoded by the fnr gene is a general regulatory protein that activates

Implication for health policy/practice/research/medical education:

This project presents the construction of an engineered synthetic promoter, which can be used for alternative expression of recombinant proteins in anaerobic conditions in $E$. coli.

Copyright (c) 2014, Ahvaz Jundishapur University of Medical Sciences; Published by Kowsar Corp. This is an open-access article distributed under the terms of the Creative Commons Attribution License, which permits unrestricted use, distribution, and reproduction in any medium, provided the original work is properly cited. 
Nasr Ret al.

transcription initiation in some anaerobic promoters such as nirB under anaerobic conditions. Expression of protein under the control of this promoter is totally dependent on FNR transcription factor in the -30 and -52 region upstream to the transcription start point. The transcription start point of the nirB gene is positioned at the -23 or -24 region, related to the ATG initiation codon (7).

There are also two heptamer regions about 20 bp upstream to the FNR region (-69 and -70 upstream to transcription start point), which are binding sites of two other regulatory proteins; NarP and NarL. Mutation in NarP, NarL or both decreases nirB promoter activity; this means that NarL and NarP are cofactors of FNR in the transcription process. It has been demonstrated mutation in the NarL binding site suppresses nitrite induction whereas it has no effect on nitrate induction $(8,9)$. A synthetic nirB promoter was successfully used for in vitro expression of fragment $\mathrm{C}$ of tetanus toxin in both E. coli and Salmonella typhimurium. This promoter also shows effective in vivo inducibility, in conditions where there is normally no oxygen tension (10).

In recombinant protein expression systems, removing these inducers from culture medium result in further steps for purification and then more cost. In addition, these chemical inducers may have some side-effects on cell physiology, and since using these inducers is not applicable in vivo, the present study was performed to construct a synthetic nirB promoter, which has no responding regions for nitrite and nitrate inducers and is only able to induce the recombinant protein under low oxygen pressure or anaerobic conditions.

\section{Objectives}

For this purpose and based on the native sequence of E. coli nirB promoter, we designed a synthetic nirB promoter and constructed a plasmid that it able to produce recombinant proteins under anaerobic induction and not by chemical inducers (nitrite or nitrate) and then the promoter activity was evaluated with regarding to the expression of a heterologous gene; heat-labile toxin B subunit (LTB gene), under various conditions.

\section{Materials and Methods}

\subsection{Bacterial Strain, Enzymes and Reagents}

E. coli DH5 was used in all cloning and expression procedures. Restriction enzymes, T4 DNA ligase, Taq DNA polymerase and dNTP were purchased from Fermentas (Lithuania). Ganglioside GMI type III, standard E. coli heat-labile toxin (LT) and anti-mouse horse-raddish peroxidase (HRP) conjugate antibodies were obtained from Sigma (USA). All cloning steps such as transformation, plasmid extraction, PCR amplification procedures and restriction digestion were performed on the basis of standard protocols (11).

\subsection{PCR Amplification of LTB Gene}

The LTB gene was generated by PCR on the pUCLTB plasmid, which was constructed as a template in our previous study (12). A 375-bp fragment was amplified using a forward primer containing NcoI site (underlined) 5’- GAATTCGGATGAAccATGgATAAAAG -3’ covering ATG translation initiation codon and a reverse primer $5^{\prime}$ - CACAAGCTTCTAGTTTTCCATGATTG-3 containing Hind III site (underlined).

\subsection{Synthesis of nirB Promoter}

The nirB synthetic promoter was designed based on the nitrite reductase gene sequence (X14202 gi: 42120) and constructed by two oligomers BamnirBSD78; (5' GATCCaggtaAATTTGATgTACATCAAatggtaccccttgctgaatcgtTAAGGTaggcggtaTaagGAGGaaaaaac) and NcoNirBSD78 (5'CATGGttttttcctccttataccgcctaccttaacgattcagcaaggggtaccatttgatgtacatcaaatttacctg). The two oligomers were mixed by molar ratio at $85^{\circ} \mathrm{C}$ and cooled slowly to form a double-stranded fragment with cohesive ends of BamHI and NcoI sites at the $5^{-}$and $3^{-}$ends, respectively. On the BamnirBSD78 oligo diagram, the FNR region has been shown by underlined capital letters, the ribosome-binding site (RBS) as bold letters and -10 region as italic capital letters.

\subsection{Construction of pFSnirB78-23LTB}

By two-step cloning, the synthetic nirB promoter (nirB: BamHI- NcoI fragment) was firstly cloned in a pkk223 derivative plasmid and then the amplified LTB gene (LTB: NcoI-HindIII fragment) was cloned downstream of the promoter. This new construct was confirmed by restriction analysis and named pFSnirB78-23LTB.

\subsection{Expression of Recombinant LTB Under Aerobic and Anaerobic Condition}

The plasmid was transformed into competent E. coli and one colony of E. coli/ pFSnirB78-23LTB was cultured overnight in Luria-Bertani (LB) medium (yeast extract, peptone and $\mathrm{NaCl}$ ) supplemented with ampicillin (100 $\mathrm{ug} / \mathrm{mL}$ ) at $37 \mathrm{C}$ with vigorous shaking (220 rpm). For evaluation of promoter activity in aerobic conditions, 1/500 of overnight culture was inoculated into $5 \mathrm{~mL}$ of fresh medium containing ampicillin and incubated at $37 \mathrm{C}$ with vigorous shaking $(220 \mathrm{rpm})$ until $\mathrm{OD}_{600 \mathrm{~nm}}$ reached 0.4-0.6. Induction was carried out by adding sodium nitrite $(2.5 \mathrm{mM})$ or sodium nitrate $(20 \mathrm{mM})$, while a reaction without chemical induction was also performed; incubation was continued for 4 hours. The cells were then harvested and resuspended in $1 \mathrm{~mL}$ of PBS.

The colony forming unit (cfu) was determined by duplicate culture of $20 \mu \mathrm{L}$ of each sample, which was serially diluted. The harvested cells were lysed by freezing and thawing three times with vigorous shaking and the crude lysate was analyzed for detection and measure- 
Nasr Ret al.

ment of the produced LTB by GM1-ELISA. The recombinant LTB concentration was calculated as ng/ $10^{9} \mathrm{cfu}$. Alternatively, for the reaction under anaerobic conditions, 1/30 of the overnight culture was inoculated into a $5 \mathrm{~mL}$ screw-cap conical tube completely filled with LB medium containing ampicillin. Sodium nitrite $(2.5 \mathrm{mM})$ or sodium nitrate $(20 \mathrm{mM})$ was added to each tube as an inducer for comparison with the control, which was kept under anaerobic conditions but did not contain the inducer. After 24 hours of incubation at $37 \mathrm{C}$, the cfu was determined and rLTB measurement was performed as mentioned above.

\subsection{Ganglioside GM1 Enzyme Linked Immunosor- bent Assay (GM1 ELISA)}

The rLTB levels were determined and measured by the GM1 ELISA method as previously reported (13). Briefly, ELISA plates were coated with $5 \mu \mathrm{g}$ ganglioside GM1 type III per well in $100 \mu \mathrm{L}$ carbonate buffer, ( $\mathrm{pH} 9.6$ ) for 6 hours at room temperature. Next, the plates were blocked with PBS-0.1\% bovine serum albumin (w/v) (PBS$\mathrm{BSA})$ at $37 \mathrm{C}$ for 30 minutes. Diluted supernatants of the samples in PBS were added to each well in duplicate and after 1 hour of incubation at $37 \mathrm{C}$, the plates were washed 3 times with PBS-T (0.05\% Tween20 in PBS). AntiLTB/cholera toxin B subunit cross reactive monoclonal antibody D15-8 (kindly provided by the Pasteur Institute, Paris) and LT39 monoclonal antibody (kindly provided by Svennerholm, university of Goteborg, Sweden) were used as the first antibody and $100 \mu \mathrm{L}$ of this diluted first antibody was added to each well and the plates were incubated at $37 \mathrm{C}$ for 1 hour. The plates were then washed three times with PBS-T, and goat anti-mouse antibody conjugated with horseradish peroxidase (HRP) was added to each well. After 1 hour of incubation at room temperature followed by washing, chromogenic substrate, tetramethylbenzidine (TMB) and $\mathrm{H}_{2} \mathrm{O}_{2}$ were added to each well. The reaction was stopped by $2 \mathrm{~N} \mathrm{H}_{2} \mathrm{SO}_{4}$ after 15 minutes and absorbance was read at $450 \mathrm{~nm}$. The amount of rLTB in each sample was determined by interpolation on standard curves generated using the reference LT. Finally, production rate of rLTB was calculated for $10^{9} \mathrm{cfu}$.

\section{Results}

In this study, we constructed a new plasmid by two-step cloning as shown in Figure 1. At first, we cloned the 78 bp synthetic nirB promoter in a pKK223 derivative plasmid and then the LTB gene (375 bp) was cloned under the control of this promoter and the final plasmid was named pFSnirB78-23LTB. The second codon of the native LTB gene was changed from AAT to GAT, which altered Asn to Asp in the second amino acid of the LTB signal peptide. The plasmid was transformed into E. coli DH5 in order to measure promotor activity following restriction analysis of the plasmid.

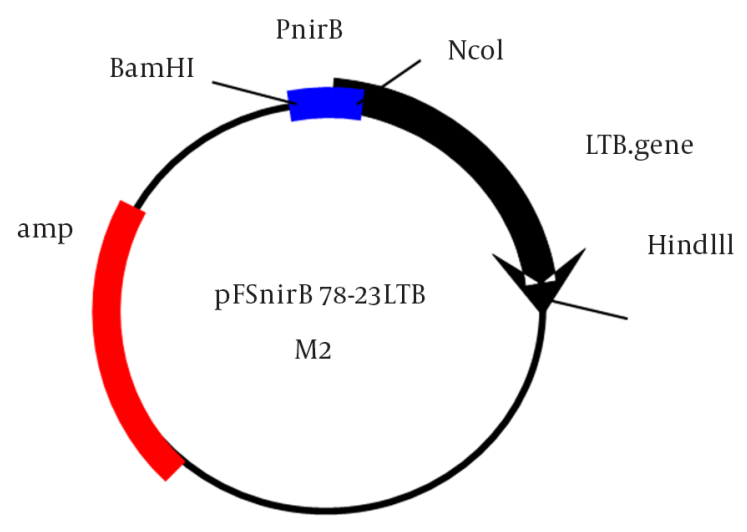

Figure 1. Schematic Construction of pFSnirB78-23LTB Plasmid

Activity of this promoter was determined by examining rLTB expression levels under both aerobic and anaerobic conditions and also the regulatory effect of chemical inducers nitrite and nitrate on the promoter was evaluated under both aerobic and anaerobic conditions. Our results showed that E. coli/ pFSnirB78-23 produces rLTB when anaerobiosis is solely the inducer. Under anaerobic conditions and also in the absence of any chemical inducer, the amount of rLTB was as high as $1560 \mathrm{ng}$ per $109 \mathrm{cfu}$ (Table 1). Chemical induction by both nitrite and nitrate had no more effect on increasing rLTB levels. Alternatively, under aerobic conditions the rLTB expression levels suppressed dramatically to as low as $11 \%$ of the anaerobic culture. However, supplementation of culture with nitrite and nitrate showed a greater decrease in rLTB expression levels.

Table 1. Comparision of rLTB Induction by E. coli/ pFSnirB7823LTB Under Aerobic and Anaerobic Induction and Evaluation of Nitrite and Nitrate on Promoter Activity

\begin{tabular}{lll}
\hline & \multicolumn{2}{l}{ Expressed rLTB, ng/10 ${ }^{9} \mathbf{c f u}$} \\
\cline { 2 - 3 } & Anaerobic & Aerobic \\
\hline Anaerobic induction & 1560 & 171 \\
Anaerobic induction + nitrite & 1600 & 79 \\
Anaerobic induction + nitrate & 1530 & 83 \\
\hline
\end{tabular}

\section{Discussion}

Chemically inducible promoters such as isopropyl-betaD-thiogalactopyranoside (IPTG) are extensively used for in vitro expression of recombinant proteins in bacteria. Due to the inducer's stability, additional time and expensive steps to remove the inducers from the final product are required. Also, these promoters are not suitable for anaerobic conditions both in vitro and in vivo where there is not enough oxygen tension for cell growth. It seems that anaerobically inducible promoters such as the nirB promoter are an attractive alternative choice as they have successfully been used by numerous investigators. Various plasmids containing synthetic nirB promot- 
Nasr Ret al.

ers have been constructed and used for expression of foreign genes in E. coli $(14,15)$. These promoters have also been used for in vivo production of various antigens by salmonella live vaccine strains (16).

Intact native nirB promoter has also been used for expression of foreign genes in Salmonella live vaccine strains and has been shown to be an efficient system for immunization (17). With the aim of dissociation of chemical induciblity from anaerobiosis, we constructed an engineered synthetic nirB promoter, and evaluated the expression of LTB gene under anaerobic conditions, and determined the regulatory effects of nitrite and nitrate as chemical inducers on the activity of this promoter. We considered some critical regulatory regions on this engineered promoter. Firstly, we considered a hexamer sequence, TAAGGT at -10 position that is necessary for anaerobic activation. It has been shown that mutation in this region may result in loss of activity of the promoter (8). Secondly, a ribosome binding site (RBS) sequence was examined for control of translation. Thirdly, we evaluated the FNR global regulatory protein-binding site at -30 and -52 , related to transcription start position. FNR protein encoded by the fnr gene regulates protein expression under anaerobic conditions via activation of transcription initiation of some anaerobic promoters such as nirB (7). We compared our nirB promoter with the intact native promoter and a synthetic promoter (pTETnir15) reported by Oxer et al. (14), as shown in Figure 2. There were some differences in the sequences that explain the probable variation between their activities.

Primarily, there were differences in the critical sequence for anaerobic activation; TAAGGT at -10 position upstream to the transcription start point and its distance to RBS. We selected this sequence so that it was exactly the same as the native promoter with slightly less distance to RBS. However, Oxer et al. preferred to choose the same sequence with more distance from RBS (14). The second critical region was the FNR protein-binding site between positions -52 and -30 , upstream of the transcription start point. In PnirB78-23, this sequence is different from the native nirB promoter with respect to the central nucleotide but it is the same as that of pTETnir15 reported by Oxer et al. However, some investigations suggest more distance may not be important in expression rate. The third critical region is the RBS sequence and its distance to ATG translation initiation codon.

We choose the TAAGGAGG sequence that it is exactly the same as the native promoter in four $3^{\prime}$ nucleotides but with more distance to the ATG codon. During the expression process, our data showed that the synthetic nirB promoter could be anaerobically induced and express rLTB in the E. coli host whereas it is extensively suppressed under aerobic conditions. Since growth of bacteria and induction of this promoter is different from other conventional expression systems, it might have adverse effects on the nature of the product. Assembling of LTB monomers in pentameric form (LTB5) is essential for binding LTB to its receptor (ganglioside GM1) and also for the immunogenicity and adjuvanticity of this molecule.

As shown by GM1-ELISA in this study, critical characteristics of the recombinant protein produced by this system such as pentamer formation, receptor recognition and antigenic epitopes are interestingly conserved. Reaction of LTB with LT39 mAb, a specific antibody for the pentamer but not the monomer form, resulted the correct structure formation of the produced LTB and conserved its receptor binding ability and immunogenic determinants. Our results are in agreement with those found by Newton and his colleagues that showed other foreign proteins could be efficiently expressed by the nirB promoter and well tolerated by E. coli (18). In E. coli FNR-dependent transcription is modulated by other regulatory systems (Nar/NarP), which appear to coordinate transcriptional responses with nitrate and nitrite. (9).

In native nirB promoter, the NarL-binding site is located at position -79 to -60 and contains an inverted repeat of two 10-base sequence elements. For removal of chemical induction from our construct, we didn't consider this element in our synthetic promoter. As we expected our promoter did not respond to chemical inducers (nitrite and nitrate) of intact native nirB promoter (9). The findings of the present study showed that synthetic nirB can anaerobically produce foreign proteins, and removing the chemical inducibility of nirB promoter from its anaerobic activity is possible. In addition, anaerobiosis has no adverse effects on the produced protein. Accordingly,

Figure 2. Comparison of Sequences of Intact Native nirB Promoter (GenBank gi:42120), pTETnir15 (14) and pFSnirB78-23 (this study)

pTETnir15........GAATTCAGGTAAATTTGATGTACATCAAATGGTACCCCTTGCTGAATCGTTAAGGTAGGCGGTA GGGCCCAGATCTTAATCATCCACAGGAGACTTTCTGATG

E, coli nirB.......

iGAATTTGATTTACATCAATAAGCGgGGTTGCTGAATCGTIAAGGTAGGCGGTAATAGAAAAGAAATCGAGGCAAA AATG

B

pFSnirB78-23 .....GATCCaggtaAATTTGATgTACATCAAatggtaccccttgctgaatcgtTAAGGTaggcggtaTaagGAGGaaaaaac

In pFSnirB78-23 plasmid the FNR region is visualized by underlined capital letters, ribosome-binding site (RBS) is shown by bold and -10 region by italic capital letters. 
Nasr Ret al.

we think the nirB promoter is a suitable system for in vitro expression especially when high oxygen tension might have destructive effects on the recombinant protein. This system is potentially suitable for in vivo delivery of antigens or drugs by live bacterial strains such as salmonella live delivery vaccine strains.

\section{Acknowledgements}

This work was supported by Semnan University of Medical Sciences. We gratefully acknowledge all the people who helped us in this study. We also thank Dr Ali Rashidipour, Dr Raheb Ghorbani and Dr Vahid Semnani for their helpful support and advice.

\section{Authors' Contributions}

Dr. Mohammad Reza Akbari developed the original idea and supervised the project. Dr. Mohammad Reza Akbari and Mr. Reza Nasr contributed to the development of all protocols, acquisition and analysis of data and preparing the manuscript.

\section{Financial Disclosure}

Dr. Mohammad Reza Akbari reported receiving financial research support from Semnan University of Medical Sciences.

\section{Funding/Support}

This study was fully supported by grant 144 from the deputy of research and technology of Semnan University of Medical Sciences.

\section{References}

1. Donovan RS, Robinson CW, Glick BR. Review: optimizing inducer and culture conditions for expression of foreign proteins under the control of the lac promoter. J Ind Microbiol. 1996;16(3):145-54.

2. Valdez-Cruz NA, Caspeta L, Perez NO, Ramirez OT, Trujillo-Roldan MA. Production of recombinant proteins in E. coli by the heat inducible expression system based on the phage lambda pL and/or pR promoters. Microb Cell Fact. 2010;9:18.

3. Sawers G, Jarsch M. Alternative regulation principles for the production of recombinant proteins in Escherichia coli. Appl Microbiol Biotechnol.1996;46(1):1-9.

4. Brautaset T, Lale R, Valla S. Positively regulated bacterial expres- sion systems. Microb Biotechnol. 2009;2(1):15-30.

5. Blazeck J, Alper HS. Promoter engineering: recent advances in controlling transcription at the most fundamental level. Biotechnol J. 2013;8(1):46-58.

6. Taylor-Robinson AW, Heal KG. Heterologous gene expression in bacterial systems under reduced oxygen tensions. Small-scale optimization precedes industrial fermentation. Prep Biochem Biotechnol. 2001;31(1):13-22.

7. Jayaraman PS, Peakman TC, Busby SJ, Quincey RV, Cole JA. Location and sequence of the promoter of the gene for the NADH-dependent nitrite reductase of Escherichia coli and its regulation by oxygen, the Fnr protein and nitrite. J Mol Biol. 1987;196(4):781-

8. Jayaraman PS, Cole JA, Busby SJ. Mutational analysis of the nucleotide sequence at the FNR-dependent nirB promoter in Escherichia coli. Nucleic Acids Res. 1989;17(1):135-45.

9. Tyson KL, Bell AI, Cole JA, Busby SJ. Definition of nitrite and nitrate response elements at the anaerobically inducible Escherichia coli nirB promoter: interactions between FNR and NarL. Mol Microbiol.1993;7(1):151-7.

10. Gomez-Duarte OG, Galen J, Chatfield SN, Rappuoli R, Eidels L, Levine MM. Expression of fragment $\mathrm{C}$ of tetanus toxin fused to a carboxyl-terminal fragment of diphtheria toxin in Salmonella typhi CVD 908 vaccine strain. Vaccine.1995;13(16):1596-602.

11. Sambrook J, Russell DW. Molecular cloning A laboratory manual. 3 th edNew York: Cold spring Harbor laboratory press; 2000.

12. Eidgahi MRA, Shahhosseiny Mh, Tabarai B, Rechinsky V. Escherichia coli heat-labile toxin B subunit: Construction of plasmids for a controlled high level production of the protein and demonstration of its immunogenicity by oral administration. Med J Islam Repub Iran. 1991;1:55-60.

13. Back E, Svennerholm AM, Holmgren J, Mollby R. Evaluation of a ganglioside immunosorbent assay for detection of Escherichia coli heat-labile enterotoxin. J Clin Microbiol. 1979;10(6):791-5.

14. Oxer MD, Bentley CM, Doyle JG, Peakman TC, Charles IG, Makoff AJ. High level heterologous expression in E. coli using the anaerobically-activated nirB promoter. Nucleic Acids Res. 1991;19(11):2889-92.

15. Ruiz-Perez F, Leon-Kempis R, Santiago-Machuca A, Ortega-Pierres G, Barry E, Levine M, et al. Expression of the Plasmodium falciparum immunodominant epitope (NANP)(4) on the surface of Salmonella enterica using the autotransporter MisL. Infect Immun. 2002;70(7):3611-20.

16. Huang Y, Hajishengallis G, Michalek SM. Induction of protective immunity against Streptococcus mutans colonization after mucosal immunization with attenuated Salmonella enterica serovar typhimurium expressing an S. mutans adhesin under the control of in vivo-inducible nirB promoter. Infect Immun. 2001;69(4):2154-61.

17. Dunstan SJ, Simmons CP, Strugnell RA. Use of in vivo-regulated promoters to deliver antigens from attenuated Salmonella enterica var. Typhimurium. Infect Immun. 1999;67(10):5133-41.

18. Newton SM, Klebba PE, Hofnung M, Charbit A. Studies of the anaerobically induced promoter pnirB and the improved expression of bacterial antigens. Res Microbiol. 1995;146(3):193-202. 